\title{
An accurate and efficient finite-difference operator for the frequency-domain wave propagation
}

\author{
Junichi TAKEKAWA $^{1}$ and Hitoshi MIKADA ${ }^{1}$ \\ ${ }^{1}$ Dept. of Civil and Earth Res. Eng., Kyoto University
}

\begin{abstract}
We newly developed a finite-difference (FD) operator for the frequency-domain acoustic wave propagation. This operator uses a stretched stencil to avoid the numerical anisotropy. In general, direct solvers for sparse matrices are used in exploration geophysical community because they have advantage over iterative ones, i.e. multi-source configuration can be simply implemented. In the frequency-domain modling, the computational costs (calculation time and computational memory) depend on not only the number of neighbors but also the bandwidth of the impedance matrix. So usage of higher-order scheme is not always conducive to the improvement of the computational costs. In the present study, we use a stretched stencil of FD operator not to increase the bandwidth in the impedance matrix. Coefficients of the stencil are determined by a minimization process. We investigate the accuracy of our new scheme using dispersion analysis and numerical experiment. They show that the proposed scheme can improve not only accuracy but also efficiency compared to the conventional 9-point scheme.
\end{abstract}

\section{INTRODUCTION}

Finite-difference method has been widely used as a simulator in many scientific and engineering fields. In recent years, full-waveform inversion (FWI) and reverse time migration (RTM) have draw attention in these fields. In FWI and RTM, inversion or imaging process requires forward modeling for calculating the theoretical wavefields or recorded waveforms. Especially in FWI, we use the difference between theoretical and observed waveforms as the minimized error function directly. So, it is important to calculate accurate forward wavefield without numerical dispersion. The main shortcoming of FWI and RTM is large amount of computational costs like calculation time and computational memory.

In the past few decades, many researchers have developed accurate and efficient finite-difference (FD) operators. In FD modeling, two types of modeling approach exist; time-domain modeling and frequency-domain modeling. In the frequency-domain modeling, there are some advantages over the time-domain modeling. One of the advantages is simple implementation of multi source based on the LU decomposition. However, increasing computational costs would become disadvantage of the frequency-domain modeling. The simple solution for this problem is to use more accurate scheme ${ }^{1), 2)}$. By using more accurate scheme, we can decrease the number of total grid points in the model. However, higher order scheme is not necessarily relevant to decrease the computational costs, because higher order scheme increase the bandwidth of the impedance matrix ${ }^{3)}$. So it is important to improve accuracy without increasing the bandwidth.

In the present study, we propose a new FD scheme to calculate accurate wavefield efficiently. To achieve accurate and efficient FD scheme, we adopt the elongated FD stencil. By adopting this type of stencil, we can improve accuracy without increasing the bandwidth. The ratio between horizontal and vertical grid spacing and FD coefficients are optimized to reduce dispersion error. To find the optimized parameters, we conduct dispersion analysis. Finally, we determine the most efficient scheme to improve accuracy based on minimization of the dispersion error.

\section{METHOD}

In this chapter, we explain our strategy of accurate and efficient finite-difference strategy. In the present study, we solve the two-dimensional Helmholtz equation as shown below.

$\frac{\partial^{2}}{\partial \mathrm{x}^{2}} \mathrm{P}+\frac{\partial^{2}}{\partial \mathrm{z}^{2}} \mathrm{P}+\frac{\omega^{2}}{\mathrm{v}^{2}} \mathrm{P}=0$

Where $\mathrm{P}$ is the pressure, $\mathrm{v}$ is the velocity, $\omega$ is the angular frequency. We apply the following finite-difference approximation for $\mathrm{x}^{-}$and 


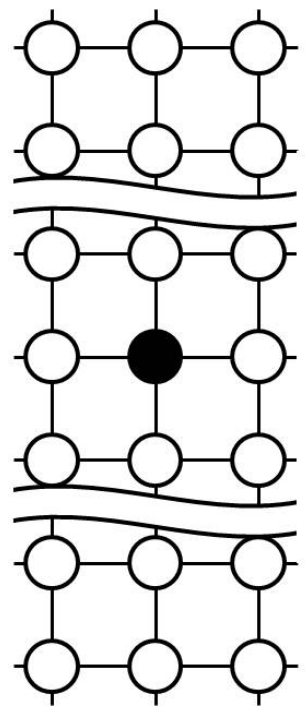

Figure 1 FD stencil in the present study. Black circle means the center of the stencil. White circles are grid points in the stencil.

z-directions, respectively.

$\frac{\partial^{2}}{\partial \mathrm{x}^{2}} \mathrm{P} \approx \frac{1}{\Delta \mathrm{x}^{2}}\left(\overline{\mathrm{P}}_{\mathrm{i}+1, \mathrm{j}}-2 \overline{\mathrm{P}}_{\mathrm{i}, \mathrm{j}}+\overline{\mathrm{P}}_{\mathrm{i}-1, \mathrm{j}}\right)$

$\frac{\partial^{2}}{\partial \mathrm{z}^{2}} \mathrm{P} \approx \frac{1}{\Delta \mathrm{z}^{2}}\left\{\mathrm{~d}_{0} \widetilde{\mathrm{P}}_{\mathrm{i}, \mathrm{j}}+\sum_{\mathrm{m}=1}^{\mathrm{M}} \mathrm{d}_{\mathrm{m}}\left(\widetilde{\mathrm{P}}_{\mathrm{i}, \mathrm{j}+\mathrm{m}}+\widetilde{\mathrm{P}}_{\mathrm{i}, \mathrm{j}-\mathrm{m}}\right)\right\}$

Where subscripts $i$ and $j$ mean indices of lattice, $M$ means the order of approximation, $\mathrm{d}_{\mathrm{m}}$ is the coefficients for FD method. In our approach, high-order approximation is applied to z-direction whereas lower-order approximation is applied to $\mathrm{x}$-direction. So, our FD stencil becomes as shown in Figure 1. $\overline{\mathrm{P}}_{\mathrm{i}, \mathrm{j}}$ and $\widetilde{\mathrm{P}}_{\mathrm{i}, \mathrm{j}}$ are weighted average of the pressure field as shown below ${ }^{4}$.

$\overline{\mathrm{P}}_{\mathrm{i}+\mathrm{k}, \mathrm{j}}=\mathrm{a}_{1} \mathrm{P}_{\mathrm{i}+\mathrm{k}, \mathrm{j}}+\sum_{\mathrm{m}=1}^{\mathrm{M}} \mathrm{a}_{\mathrm{m}+1}\left(\mathrm{P}_{\mathrm{i}+\mathrm{k}, \mathrm{j}+\mathrm{m}}+\right.$

$\mathrm{Pi}+\mathrm{k}, \mathrm{j}-\mathrm{m}$

$\widetilde{P}_{i, j+k}=b_{2} P_{i+1, j+k}+b_{1} P_{i, j+k}+b_{2} P_{i-1, j+k}$

Where $a_{m}$ and $b_{n}$ are coefficients of the weighted average in $\mathrm{z}-$ and $\mathrm{x}$-directions, respectively. By inserting equations (4) and (5), the following approximations can be obtained.

$\frac{\partial^{2}}{\partial \mathrm{x}^{2}} \mathrm{P} \approx$

$\frac{1}{\Delta \mathrm{x}^{2}}\left\{\mathrm{a}_{1}\left(\mathrm{P}_{\mathrm{i}+1, \mathrm{j}}-2 \mathrm{P}_{\mathrm{i}, \mathrm{j}}+\mathrm{P}_{\mathrm{i}-1, \mathrm{j}}\right)+\right.$

$\mathrm{m}=1 \mathrm{Mam}+1 \mathrm{Pi}+1, \mathrm{j}+\mathrm{m}+\mathrm{Pi}+1, \mathrm{j}-\mathrm{m}-2 \mathrm{Pi}, \mathrm{j}+\mathrm{m}-2 \mathrm{Pi}$

$, j-m+P i-1, j+m+P i-1, j-m$

$\frac{\partial^{2}}{\partial \mathrm{z}^{2}} \mathrm{P} \approx \frac{1}{\Delta \mathrm{z}^{2}}\left\{\mathrm{~d}_{0}\left(\mathrm{~b}_{2} \mathrm{P}_{\mathrm{i}+1, \mathrm{j}}+\mathrm{b}_{1} \mathrm{P}_{\mathrm{i}, \mathrm{j}}+\mathrm{b}_{2} \mathrm{P}_{\mathrm{i}-1, \mathrm{j}}\right)+\right.$

$\mathrm{m}=1 \mathrm{Mdmb} 2 \mathrm{Pi}+1, \mathrm{j}+\mathrm{m}+\mathrm{b} 1 \mathrm{Pi}, \mathrm{j}+\mathrm{m}+\mathrm{b} 2 \mathrm{Pi}-1, \mathrm{j}+\mathrm{m}$

$+\mathrm{b} 2 \mathrm{Pi}+1, \mathrm{j}-\mathrm{m}+\mathrm{b} 1 \mathrm{Pi}, \mathrm{j}-\mathrm{m}+\mathrm{b} 2 \mathrm{Pi}-1, \mathrm{j}-\mathrm{m}$

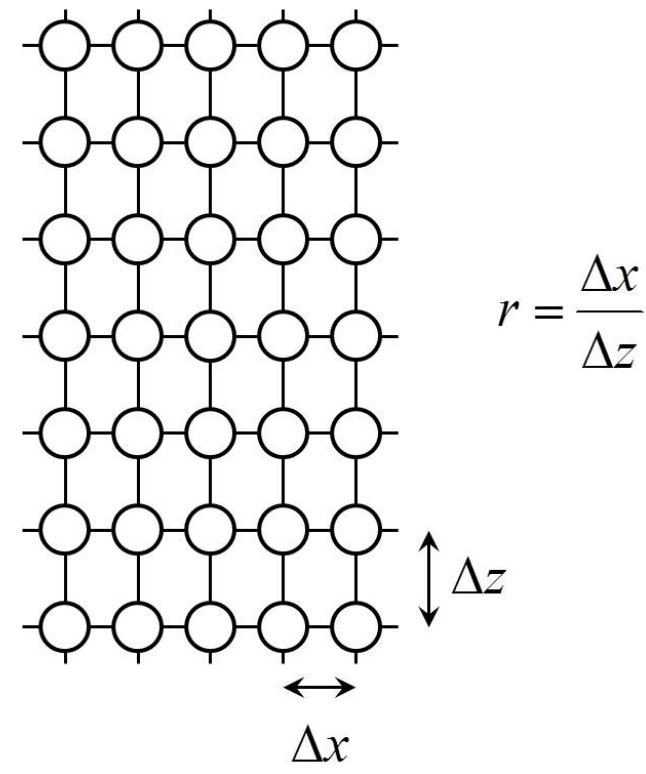

Figure 2 Elongated FD stencil.

The mass acceleration term is also divided into grid points in the stencil.

$\frac{\omega^{2}}{v^{2}} \mathrm{P} \approx$

$\frac{\omega^{2}}{v^{2}}\left\{c_{1} P_{i, j}+c_{2}\left(P_{i+1, j}+P_{i-1, j}\right)+\right.$

$\mathrm{m}=1 \mathrm{Mcm}+2 \mathrm{Pi}, \mathrm{j}+\mathrm{m}+\mathrm{Pi}, \mathrm{j}-\mathrm{m}+\mathrm{m}=1 \mathrm{Mcm}+\mathrm{M}+2 \mathrm{Pi}$

$+1, j+m+P i-1, j+m+P i+1, j-m+P i-1, j-m P$

Where $c_{m}$ are weighted coefficients. We insert equations (2) - (8) into equation (1).

$\frac{1}{\Delta \mathrm{x}^{2}}\left\{\mathrm{a}_{1}\left(\mathrm{P}_{\mathrm{i}+1, \mathrm{j}}-2 \mathrm{P}_{\mathrm{i}, \mathrm{j}}+\mathrm{P}_{\mathrm{i}-1, \mathrm{j}}\right)+\right.$

$\mathrm{m}=1 \mathrm{Mam}+1 \mathrm{Pi}+1, \mathrm{j}+\mathrm{m}+\mathrm{Pi}+1, \mathrm{j}-\mathrm{m}-2 \mathrm{Pi}, \mathrm{j}+\mathrm{m}-2 \mathrm{Pi}$

$, j-m+P i-1, j+m+P i-1, j-m+1 \Delta z 2 d 0 b 2 P i+1, j+b 1$

$\mathrm{Pi}, \mathrm{j}+\mathrm{b} 2 \mathrm{Pi}-1, \mathrm{j}+\mathrm{m}=1 \mathrm{Mdmb} 2 \mathrm{Pi}+1, \mathrm{j}+\mathrm{m}+\mathrm{b} 1 \mathrm{Pi}, \mathrm{j}+\mathrm{m}$

$+b 2 P i-1, j+m+b 2 P i+1, j-m+b 1 P i, j-m+b 2 P i-1, j$

$-m+\omega 2 v 2 c 1 P i, j+c 2 P i+1, j+P i-1, j+m=1 M c m+2$

$\mathrm{Pi}, \mathrm{j}+\mathrm{m}+\mathrm{Pi}, \mathrm{j}-\mathrm{m}+\mathrm{m}=1 \mathrm{Mcm}+\mathrm{M}+2 \mathrm{Pi}+1, \mathrm{j}+\mathrm{m}+\mathrm{Pi}-$

$1, j+m+P i+1, j-m+P i-1, j-m=0$

We determine the coefficients for the average derivatives and mass acceleration term in the next session.

\section{DISPERSION PROPERTY}

We investigate the dispersion property of the proposed method using a plane wave $\mathrm{P}=$ $\exp (\mathrm{ik} \cdot \mathbf{x}) . \quad \mathbf{k}=\left(\mathrm{k}_{\mathrm{x}}, \mathrm{k}_{\mathrm{z}}\right)$ is the wavenumber 
vector. We insert the plane wave equation into equations (6) and (7).

$\frac{\partial^{2}}{\partial \mathrm{x}^{2}} \mathrm{P} \approx$

$\frac{1}{\Delta \mathrm{x}^{2}}\left\{\mathrm{a}_{1}\left(\exp \left(\mathrm{k}_{\mathrm{x}} \Delta \mathrm{x}\right)-2+\exp \left(-\mathrm{k}_{\mathrm{x}} \Delta \mathrm{x}\right)\right) \mathrm{P}+\right.$

$\sum_{\mathrm{m}=1}^{\mathrm{M}} \mathrm{a}_{\mathrm{m}+1}\left(\exp \left(\mathrm{k}_{\mathrm{x}} \Delta \mathrm{x}\right) \exp \left(\mathrm{mk}_{\mathrm{z}} \Delta \mathrm{z}\right)+\right.$

$\exp \left(\mathrm{k}_{\mathrm{x}} \Delta \mathrm{x}\right) \exp \left(-\mathrm{mk}_{\mathrm{z}} \Delta \mathrm{z}\right)-2 \exp \left(\mathrm{mk}_{\mathrm{z}} \Delta \mathrm{z}\right)-$

$2 \exp \left(-\mathrm{mk}_{\mathrm{z}} \Delta \mathrm{z}\right)+\exp \left(-\mathrm{k}_{\mathrm{x}} \Delta \mathrm{x}\right) \exp \left(\mathrm{mk}_{\mathrm{z}} \Delta \mathrm{z}\right)+$

$\left.\left.\exp \left(-\mathrm{k}_{\mathrm{x}} \Delta \mathrm{x}\right) \exp \left(-\mathrm{mk}_{\mathrm{z}} \Delta \mathrm{z}\right)\right)\right\} \mathrm{P}$

$\frac{\partial^{2}}{\partial \mathrm{z}^{2}} \mathrm{P} \approx$

$\frac{1}{\Delta \mathrm{z}^{2}}\left\{\mathrm{~d}_{0}\left(\mathrm{~b}_{2} \exp \left(\mathrm{k}_{\mathrm{x}} \Delta \mathrm{x}\right)+\mathrm{b}_{1}+\mathrm{b}_{2} \exp \left(-\mathrm{k}_{\mathrm{x}} \Delta \mathrm{x}\right)\right)+\right.$

$\sum_{\mathrm{m}=1}^{\mathrm{M}} \mathrm{d}_{\mathrm{m}}\left(\mathrm{b}_{2} \exp \left(\mathrm{k}_{\mathrm{x}} \Delta \mathrm{x}\right) \exp \left(\mathrm{mk}_{\mathrm{z}} \Delta \mathrm{z}\right)+\right.$

$\mathrm{b}_{1} \exp \left(\mathrm{mk}_{\mathrm{z}} \Delta \mathrm{z}\right)+\mathrm{b}_{2} \exp \left(-\mathrm{k}_{\mathrm{x}} \Delta \mathrm{x}\right) \exp \left(\mathrm{mk}_{\mathrm{z}} \Delta \mathrm{z}\right)+$ $\mathrm{b}_{2} \exp \left(\mathrm{k}_{\mathrm{x}} \Delta \mathrm{x}\right) \exp \left(-\mathrm{mk}_{\mathrm{z}} \Delta \mathrm{z}\right)+\mathrm{b}_{1} \exp \left(-\mathrm{mk}_{\mathrm{z}} \Delta \mathrm{z}\right)+$

$\left.\left.\mathrm{b}_{2} \exp \left(-\mathrm{k}_{\mathrm{x}} \Delta \mathrm{x}\right) \exp \left(-\mathrm{mk}_{\mathrm{z}} \Delta \mathrm{z}\right)\right)\right\} \mathrm{P}$

Here, we define the following symbols.

$\mathrm{E}_{\mathrm{x}}=\cos \left(\mathrm{k}_{\mathrm{x}} \Delta \mathrm{x}\right)$

$\mathrm{E}_{\mathrm{mz}}=\cos \left(\mathrm{mk}_{\mathrm{z}} \Delta \mathrm{z}\right)$

Using equations (12) and (13), we rewrite equations (10) and (11).

$\frac{\partial^{2}}{\partial \mathrm{x}^{2}} \mathrm{P} \approx \frac{1}{\Delta \mathrm{x}^{2}}\left\{\mathrm{a}_{1}\left(2 \mathrm{E}_{\mathrm{x}}-2\right)+\sum_{\mathrm{m}=1}^{\mathrm{M}} \mathrm{a}_{\mathrm{m}+1}\left(4 \mathrm{E}_{\mathrm{x}} \mathrm{E}_{\mathrm{mz}}-\right.\right.$

$\left.\left.4 \mathrm{E}_{\mathrm{mz}}\right)\right\} \mathrm{P}$

$\frac{\partial^{2}}{\partial \mathrm{z}^{2}} \mathrm{P} \approx$

$\frac{1}{\Delta \mathrm{z}^{2}}\left\{\mathrm{~d}_{0}\left(2 \mathrm{~b}_{2} \mathrm{E}_{\mathrm{x}}+\mathrm{b}_{1}\right)+\sum_{\mathrm{m}=1}^{\mathrm{M}} \mathrm{d}_{\mathrm{m}}\left(4 \mathrm{~b}_{2} \mathrm{E}_{\mathrm{x}} \mathrm{E}_{\mathrm{mz}}+\right.\right.$

$\left.\left.2 \mathrm{~b}_{1} \mathrm{E}_{\mathrm{mz}}\right)\right\} \mathrm{P}$

We also insert the plane wave equation into equation (8).

$\frac{\omega^{2}}{\mathrm{v}^{2}} \mathrm{P} \approx \frac{\omega^{2}}{\mathrm{v}^{2}}\left\{\mathrm{c}_{1}+2 \mathrm{c}_{2} \mathrm{E}_{\mathrm{x}}+\sum_{\mathrm{m}=1}^{\mathrm{M}} 2 \mathrm{c}_{\mathrm{m}+2} \mathrm{E}_{\mathrm{mz}}+\right.$

$\left.\sum_{\mathrm{m}=1}^{\mathrm{M}} 4 \mathrm{c}_{\mathrm{m}+\mathrm{M}+2} \mathrm{E}_{\mathrm{x}} \mathrm{E}_{\mathrm{mz}}\right\} \mathrm{P}$

Inserting equations (14), (15) and (16) into equation (1) leads to the phase velocity of our scheme.

$\frac{1}{\mathrm{v}} \frac{\omega}{\mathrm{k}}=\frac{\mathrm{v}_{\text {num }}}{\mathrm{v}}=\frac{1}{\mathrm{k}} \sqrt{-\frac{\mathrm{A}+\mathrm{B}}{\mathrm{C}}}$

$\mathrm{A}=\frac{1}{\Delta \mathrm{x}^{2}}\left\{\mathrm{a}_{1}\left(2 \mathrm{E}_{\mathrm{x}}-2\right)+\sum_{\mathrm{m}=1}^{\mathrm{M}} \mathrm{a}_{\mathrm{m}+1}\left(4 \mathrm{E}_{\mathrm{x}} \mathrm{E}_{\mathrm{mz}}-\right.\right.$

$\left.\left.4 \mathrm{E}_{\mathrm{mz}}\right)\right\}$

$\mathrm{B}=\frac{1}{\Delta \mathrm{z}^{2}}\left\{\mathrm{~d}_{0}\left(2 \mathrm{~b}_{2} \mathrm{E}_{\mathrm{x}}+\mathrm{b}_{1}\right)+\sum_{\mathrm{m}=1}^{\mathrm{M}} \mathrm{d}_{\mathrm{m}}\left(4 \mathrm{~b}_{2} \mathrm{E}_{\mathrm{x}} \mathrm{E}_{\mathrm{mz}}+\right.\right.$

$\left.\left.2 \mathrm{~b}_{1} \mathrm{E}_{\mathrm{mz}}\right)\right\}$

$\mathrm{C}=$

$\mathrm{c}_{1}+2 \mathrm{c}_{2} \mathrm{E}_{\mathrm{x}}+\sum_{\mathrm{m}=1}^{\mathrm{M}} 2 \mathrm{c}_{\mathrm{m}+2} \mathrm{E}_{\mathrm{mz}}+$

$\sum_{\mathrm{m}=1}^{\mathrm{M}} 4 \mathrm{c}_{\mathrm{m}+\mathrm{M}+2} \mathrm{E}_{\mathrm{X}} \mathrm{E}_{\mathrm{mz}}$
Where $\mathrm{v}_{\text {num }}$ and $\mathrm{v}$ are the numerical and exact phase velocity, respectively.

For determining the coefficients and the ratio r, we define an error function $\mathrm{E}$ as shown below.

$E=\iint\left(1-\frac{v_{\text {num }}\left(\widetilde{k}, \theta ; a_{1} \sim a_{M}, b_{1}, c_{1} \sim c_{2 M+2}, r\right)}{v}\right)^{2} d \tilde{k} d \theta$

$\tilde{\mathrm{k}}=\mathrm{k} \Delta / 2 \pi, \Delta$ is a representative grid spacing $(\Delta=\sqrt{\Delta \mathrm{x} \Delta \mathrm{z}}) \cdot \mathrm{r}(=\Delta \mathrm{x} / \Delta \mathrm{z})$ is the ratio of grid spacing in horizontal and vertical directions, respectively (Figure 2). We use a hybrid approach between the simulated annealing method and the conjugate gradient method in order to optimize equation (18).

Figure 3 shows the dispersion curves for 15-point, 21-point and 27-point schemes. These schemes correspond to $\mathrm{M}=2,3$ and 4, respectively. As shown in Figure 3, the dispersion error could be suppressed by using higher schemes.

\section{CONCLUSIONS}

In the present study, we proposed a new FD stencil to improve the accuracy and efficiency of forward modeling. We investigated the effectiveness of the proposed scheme using the dispersion analysis. Dispersion curves for different order schemes show that the elongated scheme can reduce the dispersion error.

ACKNOWLEDGMENT: This work was supported by MEXT/JSPS KAKENHI (grant no. 15K18301).

\section{REFERENCES}

1) Jo, C. H., C. Shin, and J. H. Suh, 1996, An optimal 9-point, finite-difference, frequency-space, 2-D scalar wave extrapolator: Geophysics, 61(2), 529-537.

2) Shin, C., and H. Sohn, 1998, A frequency-space 2-D scalar wave extrapolator using extended 25-point finite-difference operator: Geophysics, 63(1), 289-296.

3) Tang, X., H. Liu, H. Zhang, L. Liu, and Z. Wang, 2015, An adaptable 17-point scheme for high-accuracy frequency-domain acoustic wave modeling in 2D constant density media: Geophysics, 80(6), T211-T221.

4) Chen, J. B., An average-derivative optimal scheme for frequency-domain scalar wave equation: Geophysics, 77(6), T201-T210. 

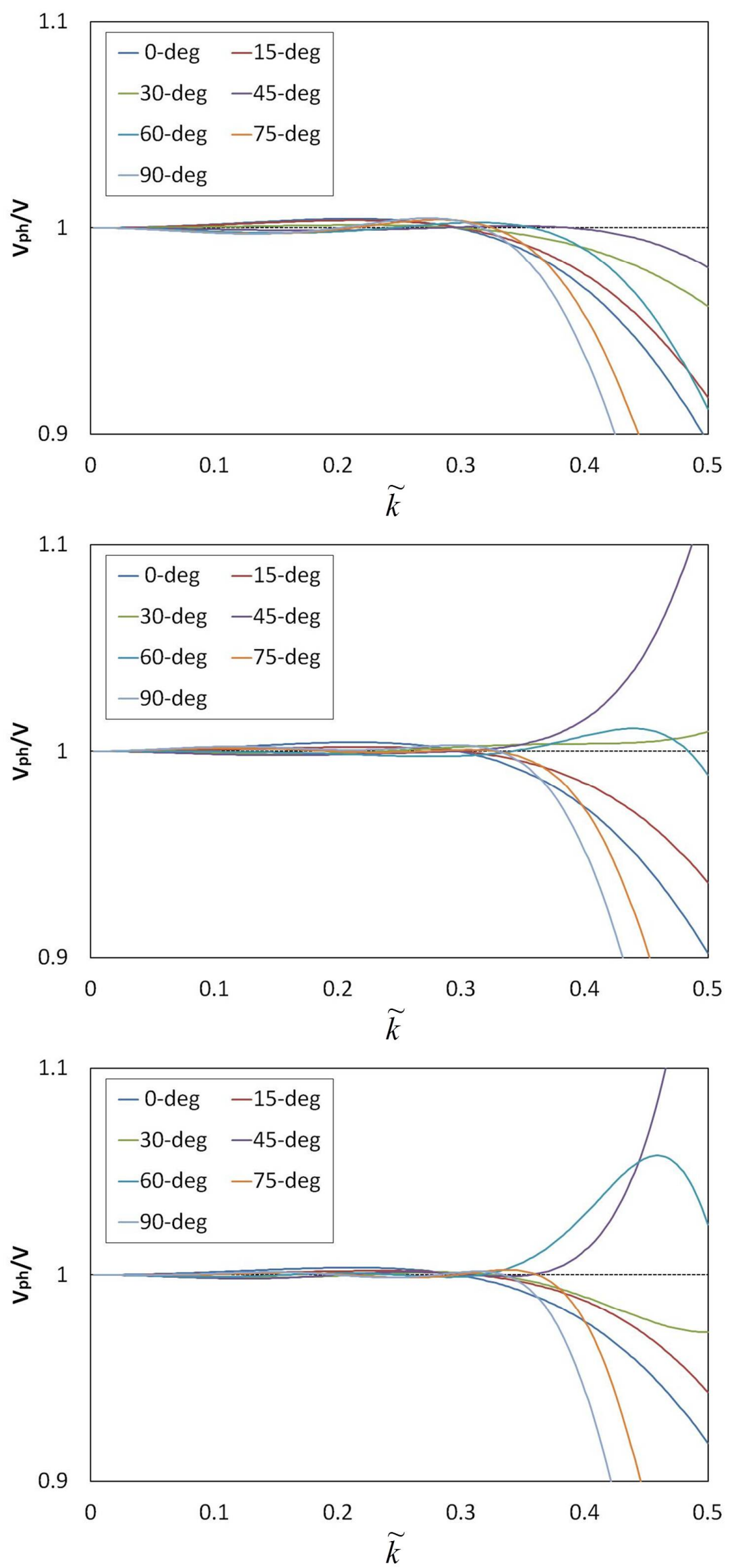

Figure 3 Dispersion curves for 15-point, 21-point and 27-point schemes with different propagating angles. 